\title{
DRP-1 functions independently of mitochondrial structural perturbations to facilitate $\mathrm{BH} 3$ mimetic-mediated apoptosis
}

\author{
Mateus Milani ${ }^{1}$, Alison J. Beckett ${ }^{2}$, Aoula Al-Zebeeby' ${ }^{1}$ Xu Luo ${ }^{3}$, lan A. Prior ${ }^{2}$, Gerald M. Cohen ${ }^{1,4}$ and \\ Shankar Varadarajan ${ }^{1,4}$
}

\begin{abstract}
Maintenance of mitochondrial integrity is critical for normal cellular homoeostasis. Most cells respond to stress stimuli and undergo apoptosis by perturbing mitochondrial structure and function to release proteins, such as cytochrome $c$, which are essential for the execution of the intrinsic apoptotic cascade. Cancer cells evade these events by overexpressing the anti-apoptotic BCL-2 family of proteins on mitochondrial membranes. Inhibitors of the antiapoptotic BCL-2 family proteins, also known as BH3 mimetics, antagonise the pro-survival functions of these proteins and result in rapid apoptosis. Although the precise mechanism by which $\mathrm{BH} 3$ mimetics induce apoptosis has been well characterised, not much is known in terms of the structural changes that occur in mitochondria during apoptosis. Using a panel of highly selective $\mathrm{BH} 3$ mimetics and a wide range of cell lines, we demonstrate that $\mathrm{BH} 3$ mimetics induce extensive mitochondrial fission, accompanied by swelling of the mitochondrial matrix and rupture of the outer mitochondrial membrane. These changes occur in a BAX/ BAK-dependent manner. Although a major mitochondrial fission GTPase, DRP-1, has been implicated in mitochondrial apoptosis, our data demonstrate that DRP-1 might function independently/downstream of $\mathrm{BH} 3$ mimetic-mediated mitochondrial fission to facilitate the release of cytochrome $c$ and apoptosis. Moreover, downregulation of DRP-1 prevented cytochrome $c$ release and apoptosis even when OPA1, a protein mediating mitochondrial fusion, was silenced. Although BH3 mimetic-mediated displacement of BAK and other BH3-only proteins from BCL-X and MCL-1 was unaffected by DRP-1 downregulation, it prevented BAK activation significantly, thus placing DRP-1 as one of the most critical players, along with BAX and BAK, that governs $\mathrm{BH} 3$ mimetic-mediated cytochrome $\mathrm{c}$ release and apoptosis.
\end{abstract}

\section{Introduction}

Most chemotherapeutic agents kill cancer cells by executing the intrinsic apoptotic pathway, which is characterised by mitochondrial outer membrane permeabilization (MOMP), release of cytochrome $c$ from the inner

\footnotetext{
Correspondence: Shankar Varadarajan (svar@liv.ac.uk)

${ }^{1}$ Department of Molecular and Clinical Cancer Medicine, Institute of

Translational Medicine, University of Liverpool, Liverpool, Ashton Street, Liverpool L69 3GE, UK

${ }^{2}$ Department of Cellular and Molecular Physiology, Institute of Translational Medicine, University of Liverpool, Liverpool, Ashton Street, Liverpool L69 3GE, UK

Full list of author information is available at the end of the article.

Edited by I. Amelio
}

mitochondrial membrane (IMM) and formation of the apoptosome that activates the initiator and effector caspases. MOMP is regulated by the BCL-2 family, whereby BAX and BAK, undergo specific conformational changes to form oligomeric pores that insert into the outer mitochondrial membrane (OMM) to release cytochrome $c^{1,2}$. Activation of BAX and BAK is achieved by several pro-apoptotic $\mathrm{BH} 3$-only members, which are generally rendered ineffective by sequestration with specific antiapoptotic BCL-2 family of proteins, such as BCL-2, BCL$\mathrm{X}_{\mathrm{L}}$ and $\mathrm{MCL}-1^{3,4}$. These anti-apoptotic proteins are highly expressed in many cancers and inhibitors known as BH3 mimetics have been designed to target them in order

\section{(c) 2019 The Author(s).}

(c) (i) Open Access This article is licensed under a Creative Commons Attribution 4.0 International License, which permits use, sharing, adaptation, distribution and reproduction c. in any medium or format, as long as you give appropriate credit to the original author(s) and the source, provide a link to the Creative Commons license, and indicate if changes were made. The images or other third party material in this article are included in the article's Creative Commons license, unless indicated otherwise in a credit line to the material. If material is not included in the article's Creative Commons license and your intended use is not permitted by statutory regulation or exceeds the permitted use, you will need to obtain permission directly from the copyright holder. To view a copy of this license, visit http://creativecommons.org/licenses/by/4.0/. 
to displace the BH3-only proteins, activate BAX and BAK, thereby inducing MOMP and apoptosis of cancer cells ${ }^{5}$.

ABT-737, and its orally available analogue, ABT-263 (Navitoclax) were the first bona fide BH3 mimetics developed to target BCL-2, BCL- $\mathrm{X}_{\mathrm{L}}$ and BCL-w ${ }^{6,7}$. Subsequently, $\mathrm{BH} 3$ mimetics that specifically target $\mathrm{BCL}-2$ (ABT-199 (Venetoclax), S55746), BCL-X $\mathrm{L}_{\mathrm{L}}$ (A-1331852) and MCL-1 (A-1210477, S63845, AMG 176 and AZD5991) have been synthesised ${ }^{8-14}$. These inhibitors, as single agents, have demonstrated much promise in treating a wide variety of haematological malignancies, and have had limited success in combination with conventional chemotherapy against several solid tumours ${ }^{8,12-17}$. BH3 mimetics induce apoptosis primarily by targeting protein-protein interactions between the anti- and proapoptotic BCL-2 family members ${ }^{18}$. Subsequently, BH3 mimetics have been shown to induce significant structural changes in the mitochondria, ranging from mitochondrial matrix swelling to discontinuities in the OMM, upstream of caspase activation ${ }^{19,20}$. Furthermore, BAX and BAK localise to the breakpoints in OMM and may facilitate cytochrome $c$ release at such breakpoints ${ }^{19}$. Although BCL-2 family members have been implicated in regulating mitochondrial membrane dynamics and functions ${ }^{21-24}$, putative inhibitors of MCL-1 have often resulted in extensive mitochondrial fission in various cell lines ${ }^{25-27}$. The regulation of this fission and its relationship to $\mathrm{BH} 3$ mimetic-mediated apoptosis remains to be determined.

Mitochondrial structure is maintained through an intricate balance between the activities of several fusion and fission proteins, which belong to a conserved family of GTPases that reside in the OMM or IMM. Mitofusins 1 and 2 (MFN1/2) and optic atrophy 1 (OPA1) are essential for mitochondrial fusion, whereas dynamin related protein 1 (DRP-1) is essential for mitochondrial fission ${ }^{28}$. Defects in mitochondrial fusion and fission have been implicated in a range of pathophysiological conditions including poor brain development, optic atrophy, cardiomyopathy and neurodegenerative diseases ${ }^{29,30}$. Mounting evidence now suggests the involvement of several members of BCL-2 family members, particularly MCL-1, in the regulation of mitochondrial structure and function $^{22-25,29}$. However, the mechanism by which MCL-1 regulates mitochondrial membrane dynamics and the potential cross-talk with its conventional role in antagonising apoptosis remain to be characterised.

In this study, we use a panel of highly selective $\mathrm{BH} 3$ mimetics together with cell lines that depend on specific BCL-2 family members for survival to demonstrate that BH3 mimetics induce significant ultrastructural mitochondrial changes upstream of caspase activation. DRP-1 plays a role downstream of these changes but upstream of MOMP to facilitate cytochrome $c$ release and apoptosis, following exposure to $\mathrm{BH} 3$ mimetics.

\section{Results}

BH3 mimetics induce marked mitochondrial structural changes

Previously, we have reported that $\mathrm{BH} 3$ mimetics induce a novel paradigm of apoptosis characterised by marked ultrastructural changes in the mitochondria, involving the loss of mitochondrial cristae and the appearance of breaks in the OMM, resulting from mitochondrial matrix swelling ${ }^{19,20,31}$. In cell lines that depend for survival almost exclusively on BCL-2 (MAVER-1), BCL- $\mathrm{X}_{\mathrm{L}}-(\mathrm{K} 562)$ and MCL-1 (H929) ${ }^{32}$, exposure to the relevant BH3 mimetics, such as ABT-199, A-1331852 and A-1210477, respectively, resulted in similar mitochondrial matrix swelling and rupture of the OMM (Fig. 1a-c). Such mitochondrial changes were also evident in H1299 cells following exposure to a combination of A-1331852 and A-1210477, as these cells depend on both BCL- $\mathrm{X}_{\mathrm{L}}$ and MCL-1 for survival (Fig. 1d). These mitochondrial ultrastructural changes were independent of effector caspases, as they were observed in cells pre-treated with Z-VAD.fmk, a broad-spectrum caspase inhibitor (Fig. 1). Exposure of the different cells to their appropriate $\mathrm{BH} 3$ mimetic resulted in mitochondrial membrane depolarisation, loss of cytochrome $c$ and induction of apoptosis, as assessed by phosphatidylserine externalisation (Supplementary Fig. S1). Exposure of the cells to Z-VAD.fmk almost completely inhibited $\mathrm{BH} 3$ mimetic-mediated apoptosis, assessed by PS externalisation, whereas little if any inhibition of cytochrome $c$ release was observed (Supplementary Fig. S1). Taken together these results suggested that the mitochondrial structural changes occurred upstream of effector caspase activation and accompanied cytochrome $c$ release, as well as a loss of mitochondrial membrane potential.

\section{BH3 mimetic-mediated mitochondrial perturbations occur in a BAX/BAK-dependent manner}

To assess whether BAX and BAK play crucial roles in BH3 mimetic-mediated ultrastructural changes in mitochondria, we exposed HCT-116 WT and BAX/BAK double knock-out (DKO) cells to a combination of A1331852 and A-1210477, as HCT-116 cells also depend on both BCL- $\mathrm{X}_{\mathrm{L}}$ and MCL-1 for survival ${ }^{33}$. Exposure of the HCT-116 WT cells to the BH3 mimetics resulted in significant mitochondrial matrix swelling accompanied by a loss of mitochondrial cristae, although rupture of the OMM was not readily apparent (Fig. 2). However, all these mitochondrial changes were clearly prevented in the HCT-116 BAX/BAK DKO cells, demonstrating a requirement for BAX and/or BAK for the perturbation of the mitochondria. Since BH3-only members are generally required to activate $\mathrm{BAX}$ and $\mathrm{BAK}$, we wished to assess whether BH3 mimetics could induce mitochondrial structural perturbations in the absence of all known pro- 

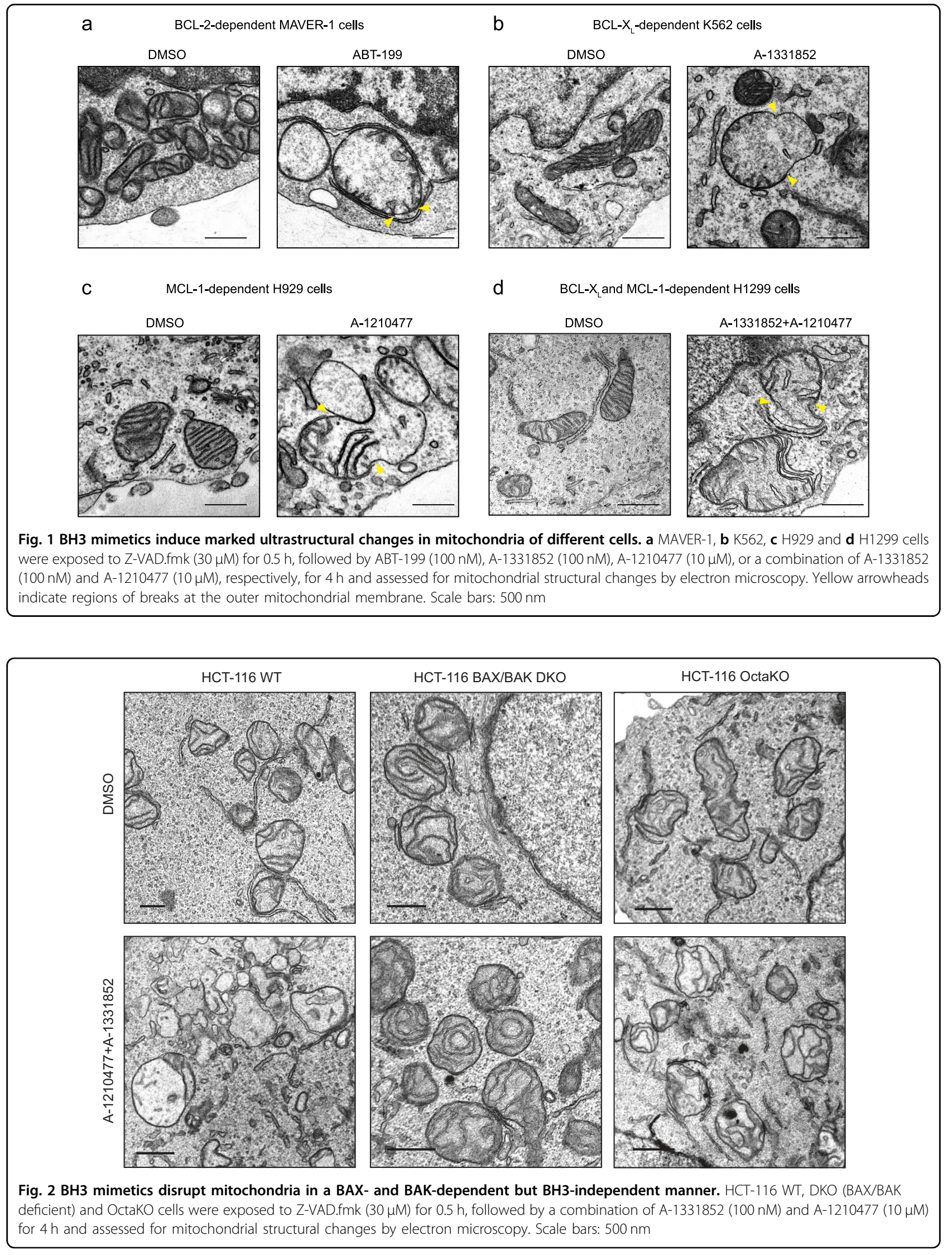


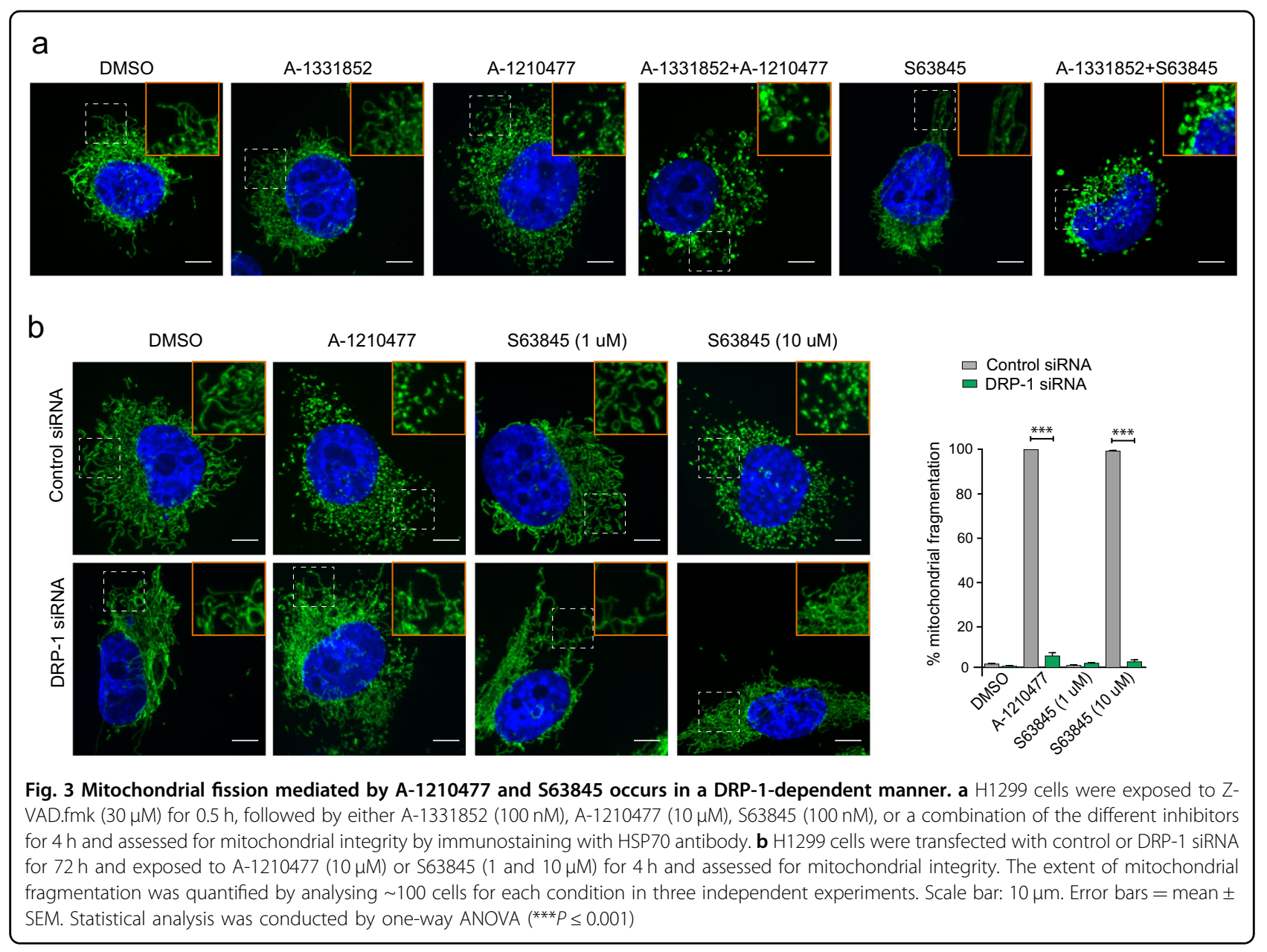

apoptotic BH3-only members. For this, we used HCT-116 OctaKO cells, which lack the BH3-only members namely, BIM, BID, PUMA, BAD, BIK, HRK, BMF and NOXA ${ }^{33}$. Exposure of these cells to a combination of A-1331852 and A-1210477 resulted in mitochondrial structural changes, characteristic of significant cristae remodelling (Fig. 2). However, the swelling of mitochondrial matrix and the accompanying loss of cristae observed in the HCT-116 WT cells following BH3 mimetics were not apparent in HCT-116 OctaKO cells (Fig. 2). This is consistent with earlier findings demonstrating that the proapoptotic BH3-only members are dispensable for $\mathrm{BH} 3$ mimetic-mediated apoptosis ${ }^{34}$. Taken together, our data demonstrated that the activation of BAX and/or BAK, either in a BH3-dependent or independent manner, is essential for the ultrastructural changes observed in the mitochondria, following exposure to $\mathrm{BH} 3$ mimetics.

\section{DRP-1 is not required for the mitochondrial structural changes that occur during the onset of apoptosis}

We previously reported that putative inhibitors of MCL-1 induced extensive mitochondrial fission and suggested that this could be a prerequisite for the ensuing apoptosis in MCL-1-dependent cell lines ${ }^{25-27}$. In support of this suggestion, exposure of A-1210477 but not A1331852 resulted in extensive mitochondrial fission that resembled mitochondrial fragmentation in H1299 cells (Fig. 3a, Supplementary Fig. S2). The ability of A-1210477 to induce mitochondrial fission was also clearly evident when used in combination with A-1331852 to induce apoptosis in these cells (Fig. 3a, Supplementary Fig. S2). However, mitochondria in this instance appeared swollen, potentially indicating swollen matrix and loss of cristae that were previously observed at the level of electron microscopy (compare Figs. 1d and 3a). In marked contrast, S63845 at a concentration $(100 \mathrm{nM})$ sufficient to induce apoptosis in a MCL-1-dependent manner ${ }^{34}$ failed to demonstrate mitochondrial fission (Fig. 3a, Supplementary Fig. S2). However, S63845 (100 nM) when used in conjunction with A-1331852 resulted in mitochondrial structural changes that resembled the swollen mitochondria observed following a combination of A-1210477 and A-1331852 (Fig. 3a, Supplementary Fig. S2). Taken together, our results suggested that mitochondrial fission 


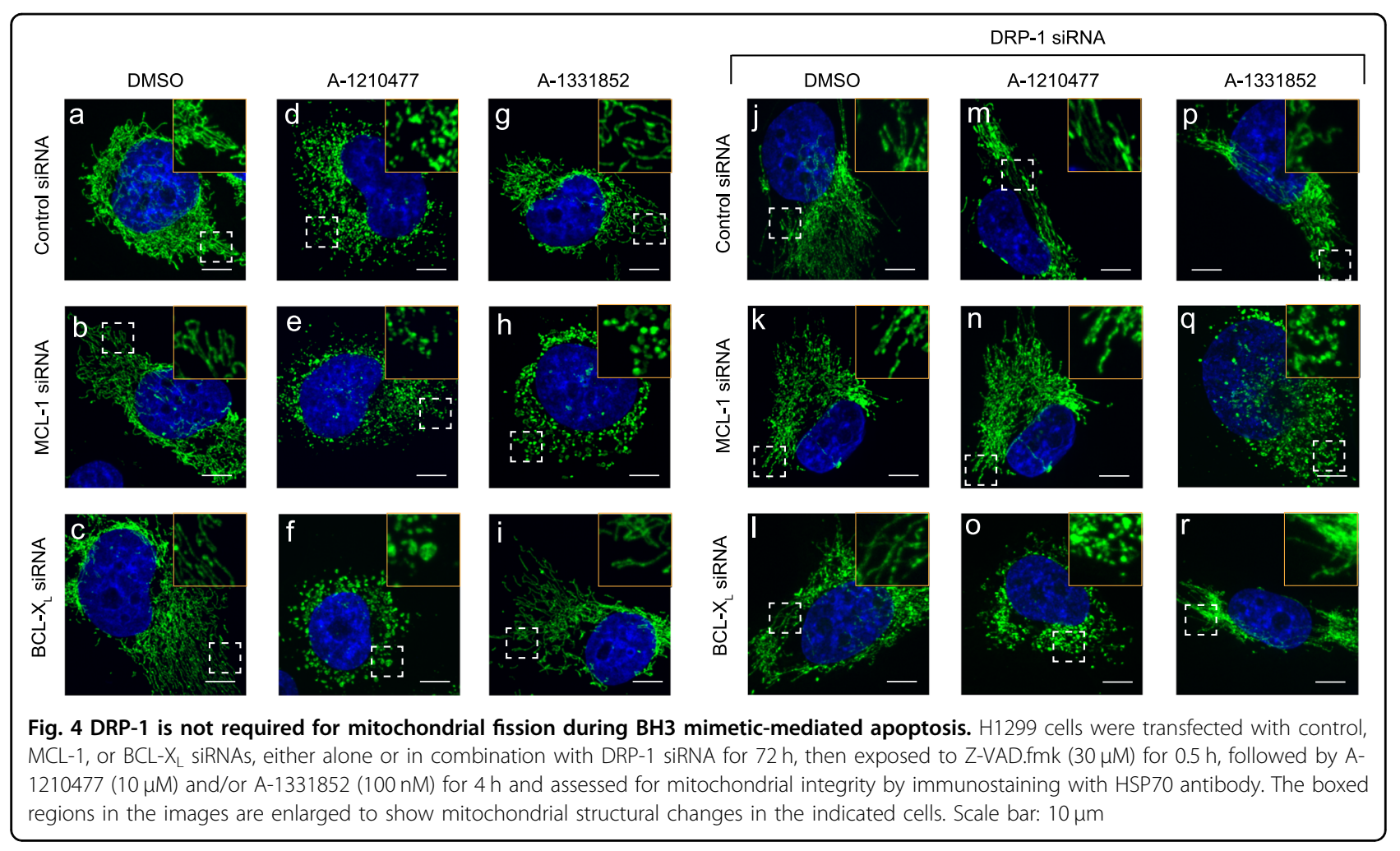

mediated by A-1210477 versus a combination of MCL-1 and $\mathrm{BCL}-\mathrm{X}_{\mathrm{L}}$ inhibitors was distinct. Moreover, while S63845 failed to exhibit mitochondrial fission at low concentrations (100-1000 nM), higher concentrations $(10 \mu \mathrm{M})$ of $\mathrm{S} 63845$ resulted in significant mitochondrial fission, which mimicked A-1210477-mediated mitochondrial fragmentation (Fig. 3b).

We previously reported that A-1210477-mediated mitochondrial fission occurred in a DRP-1-dependent manner ${ }^{27}$. A similar dependence on DRP-1 was also observed in cells exhibiting extensive mitochondrial fission, following exposure to high concentrations of S63845 (Fig. 3b). Thus both the MCL-1 inhibitors, A-1210477 and S63845, induced mitochondrial fission, which was clearly dependent on DRP-1 (Fig. 3b). We wished to assess if such mitochondrial fission was a prerequisite for apoptosis induction. Since H1299 cells depend on both BCL$\mathrm{X}_{\mathrm{L}}$ and MCL-1 for survival, we exposed cells to either A1210477 or A-1331852 and simultaneously silenced the expression levels of either $\mathrm{BCL}-\mathrm{X}_{\mathrm{L}}$ or $\mathrm{MCL}-1$ to facilitate apoptosis. Although downregulation of BCL-XL or MCL1 did not result in mitochondrial fission and maintained the filamentous structure, exposure of the cells to A1210477 resulted in significant mitochondrial fission, which resembled fragmented mitochondria (Fig. 4a-d, Supplementary Fig. S3). In the MCL-1-downregulated cells, A-1210477 still retained its ability to cause mitochondrial fragmentation (Fig. 4e), but when BCL- $\mathrm{X}_{\mathrm{L}}$ was downregulated, A-1210477 resulted in mitochondrial structural changes that resembled matrix swelling (Fig. 4f, Supplementary Fig. S3), as previously described (Fig. 1d). In contrast, exposure to A-1331852 only resulted in similar mitochondrial swelling when MCL-1 was also downregulated (Fig. 4g-I, Supplementary Fig. S3). These results suggested that mitochondrial fission, mediated by MCL-1 inhibitors, appeared to exhibit a distinct morphology from that observed following the induction of apoptosis. This was more apparent following DRP-1 downregulation, which prevented A-1210477-mediated mitochondrial fission (Fig. 4m, n), but did not appear to alter mitochondrial swelling observed during apoptosis induction (Fig. 4o, q, Supplementary Fig. S3). Taken together, these results exclude an involvement of DRP-1 in the early mitochondrial structural changes including mitochondrial swelling associated with the onset of apoptosis (Fig. 4).

Consistent with the above hypothesis, electron micrographs revealed marked structural alterations of the mitochondria in cells exposed to both A-121077 and A1331852, characterised by breaks in the OMM (denoted by the yellow arrowheads), mitochondrial matrix swelling and a concomitant loss of cristae (Fig. 5a). Downregulation of DRP-1 alone resulted in elongated mitochondria, consistent with its known role in mitochondrial fission (Fig. 5a). However, the mitochondria in the DRP-1downregulated cells following exposure to $\mathrm{BH} 3$ mimetics 


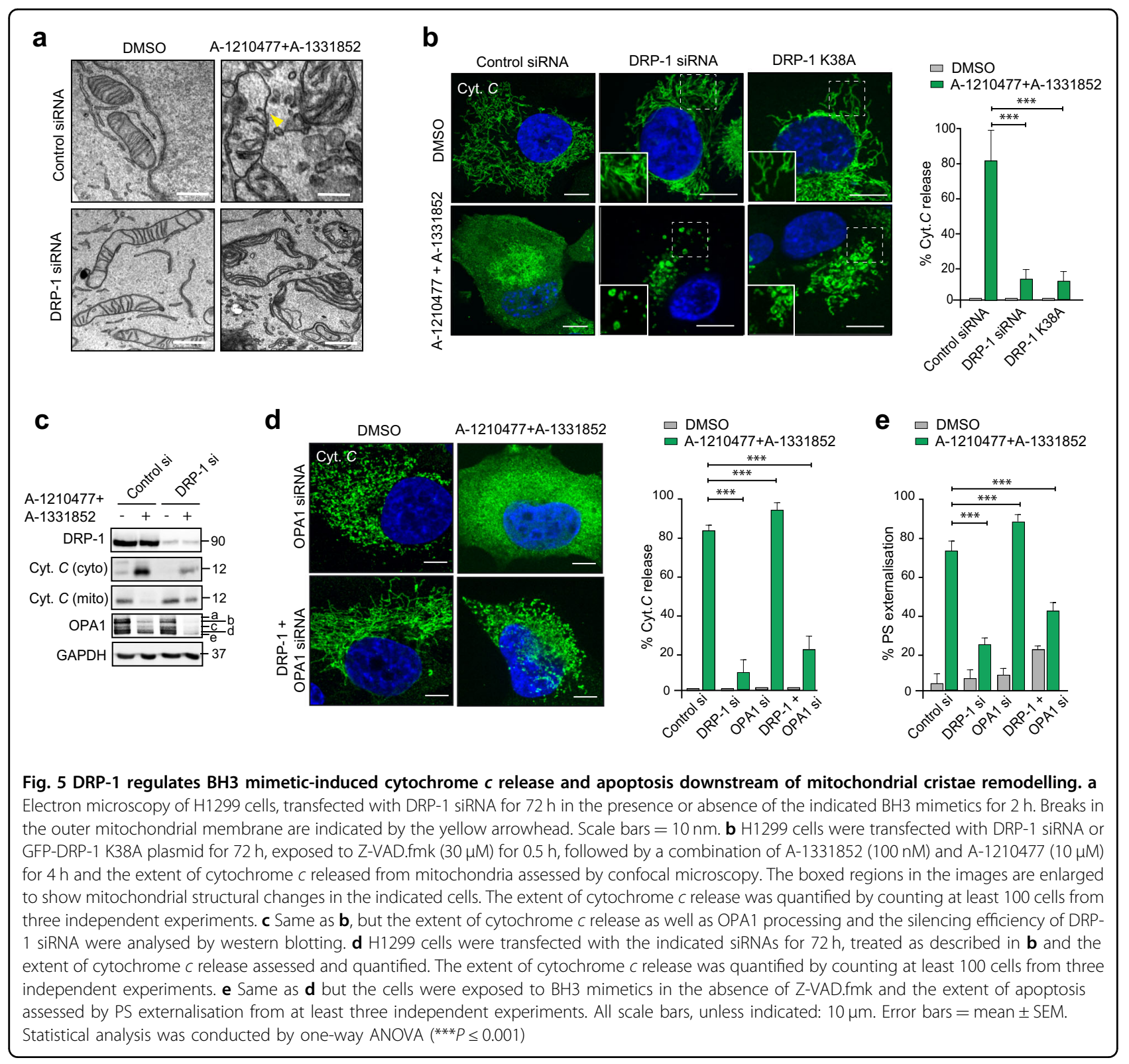

appeared visibly swollen with intact cristae and few if any breaks in the OMM (Fig. 5a). Taken together, our data suggested that mitochondrial fission observed following exposure to MCL-1 inhibitors was distinct from the structural perturbations (characterised by OMM breaks and IMM swelling) observed as a result of apoptosis induction.

\section{DRP-1 is critical for the release of cytochrome $c$ from mitochondria during apoptosis}

Permeabilisation of the OMM, otherwise known as MOMP, occurs as a consequence of BAX and/or BAK oligomerization and is generally accompanied by the release of mitochondrial cytochrome $c$ into the cytosol.
Exposure of cells to a combination of A-1210477 and A1331852 resulted in an almost complete release of mitochondrial cytochrome $c$ into the cytosol (Fig. 5b, c). This was markedly inhibited in cells, following inactivation of DRP-1 using siRNA or overexpression of the DRP-1 K38A plasmid (Fig. 5b, c), thus placing DRP-1 upstream of cytochrome $c$ release. While cytochrome $c$ was still retained in mitochondria following DRP-1 downregulation, mitochondria in these cells appeared swollen (Fig. 5b), consistent with those observed in the electron micrographs (Fig. 5a). As cytochrome $c$ release occurs as a consequence of mitochondrial cristae remodelling ${ }^{35}$, exposure to $\mathrm{BH} 3$ mimetics not only resulted in the release of mitochondrial cytochrome $c$ but also caused a loss of 
the high molecular weight isoforms of OPA1, characteristic of mitochondrial cristae remodelling (Fig. 5c). While downregulation of DRP-1 markedly diminished BH3 mimetic-mediated release of cytochrome $c$, it did not prevent BH3 mimetic-mediated loss of OPA1 (Fig. 5c), thus placing DRP-1 upstream of cytochrome $c$ release but downstream of mitochondrial cristae remodelling. This was further confirmed following exposure of DRP-1 and/ or OPA1-downregulated cells to $\mathrm{BH} 3$ mimetics. While downregulation of OPA1 resulted in significant mitochondrial fission, as well as a near-complete release of cytochrome $c$ following $\mathrm{BH} 3$ mimetics, a simultaneous downregulation of DRP-1 diminished these effects (Fig. $5 d$ ). Similarly downregulation of DRP-1 prevented BH3 mimetic-induced apoptosis, even in the absence of OPA1 (Fig. 5e), thus placing DRP-1 downstream of OPA1 proteolysis but upstream of cytochrome $c$ release in $\mathrm{BH} 3$ mimetic-mediated apoptosis.

\section{DRP-1 is critical for BAK activation during BH3 mimetic- mediated apoptosis}

Our results indicated that $\mathrm{BH} 3$ mimetics could induce structural perturbations in the mitochondria, characterised by OPA1 proteolysis, cristae remodelling and the accompanying redistribution of cytochrome $c$ from cristae to mitochondrial inner membrane space, all irrespective of the presence or absence of DRP-1. Since the role of DRP-1 was placed upstream of cytochrome $c$ release, we wished to assess whether DRP-1 impacted on any upstream events during $\mathrm{BH} 3$ mimetic-mediated apoptosis. The primary function of $\mathrm{BH} 3$ mimetics is to disrupt the protein-protein interactions between the anti-apoptotic $\left(\mathrm{BCL}-\mathrm{X}_{\mathrm{L}}\right.$ and MCL-1, in this instance) and pro-apoptotic members of the BCL-2 family. The released pro-apoptotic proteins could then activate the effector proteins (BAK, in H1299 as these cells lack BAX) to oligomerise on mitochondrial membranes to subsequently release cytochrome $c$ (Fig. 6a).

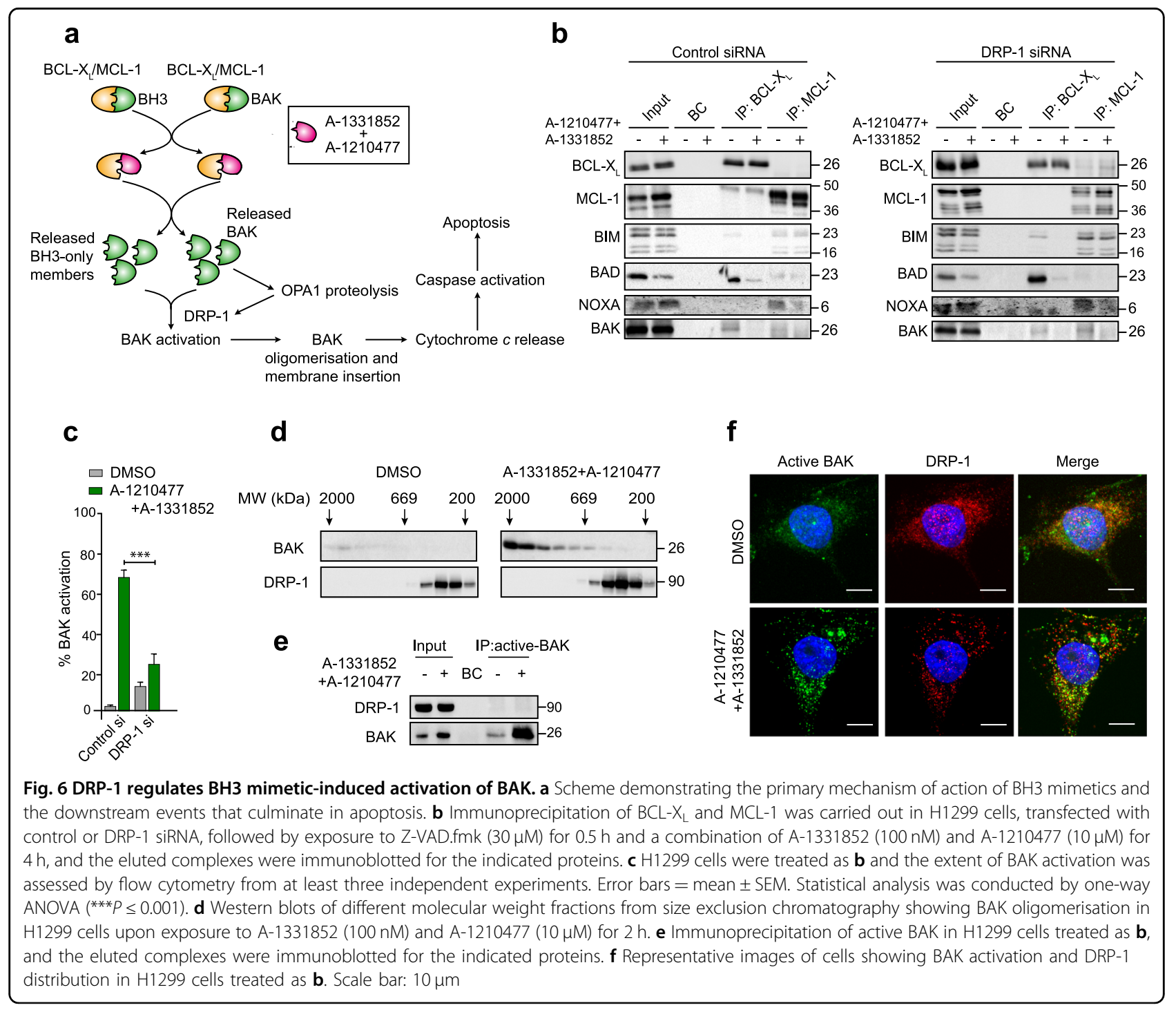


Immunoprecipitation of $\mathrm{BCL}-\mathrm{X}_{\mathrm{L}}$ and MCL-1 to identify their associated pro-apoptotic proteins revealed that in H1299 cells, BIM and BAK were bound to both BCL- $\mathrm{X}_{\mathrm{L}}$ and MCL-1, whereas NOXA and BAD exclusively bound to $\mathrm{MCL}-1$ and BCL- $\mathrm{X}_{\mathrm{L}}$, respectively (Fig. $6 \mathrm{~b}$ ). Exposure of these cells to a combination of A-1331852 and A-1210477 resulted in displacement of most of these pro-apoptotic proteins from their corresponding ant-apoptotic partners (Fig. 6b). Importantly, none of these interactions/displacements were altered in cells following DRP-1 downregulation, thus suggesting that DRP-1 played no role in the early events of BH3 mimetic-mediated apoptosis. Since BAK and other BH3-only proteins were released following BH3 mimetics, we next wished to assess if BAK activation was altered in the absence of DRP-1. Downregulation of DRP-1 resulted in a significant decrease in $\mathrm{BH} 3$ mimeticmediated activation of BAK (Fig. 6c), suggesting that DRP1 was critical in the activation of BAK during BH3 mimetic-mediated apoptosis. Although the requirement of DRP-1 for BAK activation could be demonstrated, no binding of DRP-1 to the oligomerised/active BAK was observed in these cells (Fig. $6 \mathrm{~d}-\mathrm{f}$ ), thus suggesting the involvement of other protein(s) in BAK activation immediately preceding cytochrome $c$ release. Taken together, our data confirm that DRP-1 plays a critical role at the level of BAK activation, facilitating OMM breaks, cytochrome $c$ release and apoptosis.

\section{Discussion}

BH3 mimetics, in particular ABT-737 and ABT-199, induce a novel paradigm of cell death, characterised by excessive swelling of mitochondrial matrix and discontinuities in the OMM in BCL-2-dependent chronic lymphocytic leukaemia cells ${ }^{19,31}$. BH3 mimetics targeting $\mathrm{BCL}-\mathrm{X}_{\mathrm{L}}$ and MCL-1 also induce similar mitochondrial ultrastructural changes in cells that exclusively depend on BCL- $\mathrm{X}_{\mathrm{L}}$ and MCL-1, respectively (Fig. 1$)^{20}$. However, cells exposed to the MCL-1 inhibitor, A-1210477, exhibit marked mitochondrial changes, in particular mitochondrial fission, irrespective of their dependencies on a specific BCL-2 family member for survival (Fig. 3). This is in agreement with our previous findings ${ }^{27}$. Mitochondrial fission mediated by A-1210477 alone did not result in apoptosis in these cell lines, even after prolonged exposure $^{27}$. This was most probably because most cell lines derived from solid tumours depend on both BCL- $\mathrm{X}_{\mathrm{L}}$ and MCL-1 for survival, and inhibition of MCL-1 alone was not sufficient to result in apoptosis. This was further supported by our observation that inhibition of MCL-1 using A-1210477 while resulting in extensive mitochondrial fission did not induce OMM breaks and cell death, unless the activity of BCL- $\mathrm{X}_{\mathrm{L}}$ was also neutralised.

Although A-1210477-mediated mitochondrial fission did not necessarily result in apoptosis, it was difficult to ascertain whether such fission was a prerequisite for apoptosis. This difficulty was partly because DRP-1 appeared to play important but distinct roles both in A1210477-mediated mitochondrial fission and $\mathrm{BH} 3$ mimetic-mediated apoptosis ${ }^{27}$. Moreover, DRP-1 also interacted with $\mathrm{MCL}-1$ and $\mathrm{BCL}-\mathrm{X}_{\mathrm{L}}$, thus coupling mitochondrial fission and apoptosis ${ }^{23,27,36}$. However, with the development of more potent inhibitors, such as S63845, we have demonstrated that mitochondrial fission does not occur at concentrations sufficient to inhibit MCL-1 (Fig. 3). Furthermore, while mitochondrial fission induced by A- 1210477 and high concentrations of S63845 was mediated by DRP-1, mitochondrial swelling that occurred at the onset of apoptosis induction was largely independent of DRP-1 (Figs. 3 and 4), thus differentiating the distinct types of mitochondrial fission.

Our data in the HCT-116 WT and BAX/BAK DKO cells convincingly demonstrate that $\mathrm{BH} 3$ mimeticmediated OMM breaks and swelling of matrix compartment are essential for BAX/BAK to facilitate cytochrome $c$ release (Fig. 2). The inability of HCT-116 OctaKO cells to prevent $\mathrm{BH} 3$ mimetic-mediated mitochondrial changes further supports our findings that BAX and BAK but not the known BH3-only members are critical for $\mathrm{BH} 3$ mimetic-mediated apoptosis ${ }^{33,34}$. How BAX and BAK localise to the sites of OMM breaks to facilitate cytochrome $c$ release is not entirely known. The involvement of DRP-1, Dynamin-2, and even membranes of the endoplasmic reticulum in these events have been previously proposed ${ }^{37-42}$. Downregulation of DRP-1 or its receptors, MID49 and MID51, have been shown to antagonise cytochrome $c$ release and apoptosis in response to a wide variety of apoptotic stimuli ${ }^{43}$. DRP-1 functions downstream of OPA1-mediated cristae remodelling (Fig. 5), to activate BAK (Fig. 6), which in turn precedes $\mathrm{BAK}$ oligomerisation and membrane insertion for the execution of MOMP and apoptosis. However, mitochondrial cristae remodelling requires the presence of BAX and BAK (Fig. 2) ${ }^{44}$. Thus DRP-1 could function either downstream or independent of OPA1 proteolysis to activate BAK and ensuing apoptosis. Taken together, our data suggest that $\mathrm{BH} 3$ mimetics most likely activate BAX/ BAK independently of the eight known BH3-only members, which further results in OPA1-mediated cristae remodelling to redistribute cytochrome $c$ within the mitochondria, thus priming the mitochondria to undergo MOMP, upon sensing the stress signal. DRP-1 plays a critical role at this stage to activate BAK and/or BAX to insert these effector proteins on mitochondrial membranes. This along with the constriction of the primed mitochondria by DRP-1 constitute the so-called stress signals that cause OMM breaks, efficiently releasing the redistributed cytochrome $c$ into the cytosol and initiating apoptosis. 


\section{Materials and methods \\ Cell culture}

H1299 (purchased from ATCC), K562 (provided by Prof. R. Clark, University of Liverpool) and MAVER-1 cells (provided by Dr. J. Slupsky, University of Liverpool) were cultured in RPMI 1640 medium (Life Technologies). H929 cells (purchased from DMSZ, Braunshweig, Germany) were cultured in RPMI 1640 medium supplemented with $0.05 \mathrm{mM} \beta$-mercaptoethanol (BME). Colon cancer cell lines HCT-116 (wild-type and DKO) (from R. J. Youle, National Institute of Health, USA) and HCT116-OctaKO ${ }^{33}$ were cultured in McCoy's 5A Modified media. All culture media were supplemented with $10 \%$ FBS (Life Technologies) and maintained at $37^{\circ} \mathrm{C}$ in a humidified atmosphere of $5 \% \mathrm{CO}_{2}$. All cell lines used in this study were subjected to short tandem repeat (STR) profiling to ensure quality and integrity.

\section{Reagents}

ABT-199, A-1210477 and Z-VAD.FMK from Selleck (Houston, TX, USA), S63845 from Active Biochem (Kowloon, Hong Kong) and A-1331852 from AbbVie Inc. (North Chicago, IL, USA) were used. Antibodies against HSP70 (cat\#ab2799) from Abcam (Cambridge, UK), OPA1 (cat\#612607), cytochrome $c$ (cat\#556432) and DRP-1 (cat\#611113) from BD Biosciences (San Jose, CA, USA); BCL- $\mathrm{X}_{\mathrm{L}}$ (cat\#2762), BIM (cat\#2933) and BAD (cat\#9292) from Cell Signalling Technology (MA, USA); BAK (AB-1) (cat\#AM-03) and NOXA (cat\#OP180) from Millipore (Watford, UK) and MCL-1 (cat\#sc-819), BAK (cat\#sc-832) and GAPDH (cat\#sc-25778) from Santa Cruz Biotechnologies (Santa Cruz, CA, USA) were used. All other reagents were obtained from Sigma Aldrich (St. Louis, MO, USA).

\section{Overexpression and genetic silencing}

For transient overexpression studies, cells were transfected with GFP-DRP1 K38A plasmid (provided by Dr. E. Bampton, University of Leicester, UK), using TransIT-LT1 transfection reagent (Mirus Bio LLC, Madison, WI, USA), according to the manufacturer's protocol. For RNA interference, cells were transfected with $10 \mathrm{nM}$ of siRNAs against DRP-1 (s104274235), MCL-1 (s8585 or SI02781205) or BCL- $\mathrm{X}_{\mathrm{L}}$ siRNA (s1920) purchased from Qiagen Ltd (Manchester, UK) or ThermoFisher Scientific (Waltham, MA, USA). Cells were transfected using 0.33\% (v/v) Interferin reagent (Polyplus Transfection Inc., NY) to culture media, according to the manufacturer's protocol and processed $72 \mathrm{~h}$ after transfection.

\section{Microscopy}

For electron microscopy, cells were fixed in $2.5 \%(\mathrm{w} / \mathrm{v})$ glutaraldehyde and $2 \mathrm{mM}$ calcium chloride in $0.1 \mathrm{M}$ cacodylate buffer ( $\mathrm{pH}$ 7.4). This was followed by heavy metal staining, which consisted of two consecutive osmium tetroxide steps $\left(2 \%(\mathrm{w} / \mathrm{v}) \mathrm{OsO} 4\right.$ in $\left.\mathrm{ddh}_{2} \mathrm{O}\right)$, followed by $1 \%(\mathrm{w} / \mathrm{v})$ aqueous uranyl acetate. To prevent precipitation artefacts, the cells were washed copiously with $\mathrm{ddH}_{2} \mathrm{O}$ between each staining step. All fixation and staining steps were performed in a Pelco Biowave ${ }^{\circledast}$ Pro (Ted Pella Inc., Redding, California, USA) at 100w 20Hg, for $3 \mathrm{~min}$ and $1 \mathrm{~min}$, respectively. Dehydration was in a graded ethanol series before filtration and embedding in medium premix resin (TAAB, Reading, UK). Seventy to $74 \mathrm{~nm}$ serial sections were cut using a UC6 ultra microtome (Leica Microsystems, Wetzlar, Germany) and collected on Formvar $(0.25 \%(\mathrm{w} / \mathrm{v})$ in chloroform (TAAB, Reading, UK) coated Gilder 200 mesh copper grids (GG017/C; TAAB, Reading, UK). Images were acquired on a $120 \mathrm{kV}$ Tecnai G2 Spirit BioTWIN (FEI, Hillsboro, Oregon, USA) using a MegaView III camera and analySIS software (Olympus, Germany). For immunocytochemistry, cells grown on coverslips were fixed with $4 \%(\mathrm{w} / \mathrm{v})$ paraformaldehyde, permeabilised with $0.5 \%(\mathrm{v} / \mathrm{v})$ Triton $\mathrm{X}-100$ in PBS, followed by incubations with primary antibodies (diluted 1:250 in 3\% BSA in PBS), the appropriate fluorophore-conjugated secondary antibodies (diluted 1:1000 in 3\% BSA in PBS), mounted on glass slides and imaged using a $3 \mathrm{i}$ Marianas spinning disk confocal microscope, fitted with a Plan-Apochromat $\times 63 /$ 1.4 NA oil objective, M27 and a Hamamatsu ORCAFlash4.0 v2 sCMOS Camera (all from Intelligent Imaging Innovations, $\mathrm{GmbH}$, Germany).

\section{Cytochrome $c$ release assay}

Approximately $10^{6}$ cells were washed in cold PBS and resuspended in mitochondrial isolation buffer $(250 \mathrm{mM}$ sucrose, $20 \mathrm{mM}$ HEPES, pH 7.4, $5 \mathrm{mM} \mathrm{MgCl}_{2}$ and $10 \mathrm{mM}$ $\mathrm{KCl}$ ) containing $0.01 \%$ digitonin. Cells were left on ice for $5 \mathrm{~min}$ followed by centrifugation at $13000 \mathrm{~g}$ for $3 \mathrm{~min}$ at $4{ }^{\circ} \mathrm{C}$. Subsequently, the supernatant (cytosolic fraction) and pellet (mitochondrial fraction) were processed for western blotting.

\section{Size exclusion chromatography, immunoprecipitation and western blotting}

Size exclusion chromatography and immunoprecipitation experiments were carried out as previously described $^{16,27}$. Western blotting was carried out according to standard protocols. Briefly, $50 \mu \mathrm{g}$ of total protein lysate was subjected to SDS-PAGE electrophoresis. Subsequently proteins were transferred to nitrocellulose membrane, probed with appropriate primary antibodies (diluted 1:1000 in Tris-buffered saline with $0.1 \%$ Tween-20), species-specific secondary antibodies (diluted 1:2000 in Tris-buffered saline with $0.1 \%$ Tween-20) and protein bands visualised with ECL reagents (GE Healthcare). 


\section{Flow cytometry}

The extent of apoptosis in cells following different treatments was quantified by using an Attune NxT flow cytometer (ThermoFisher Scientific, Paisley, UK) following staining of the cells with AnnexinV-FITC and propidium iodide to measure phosphatidylserine externalisation, as previously described ${ }^{45}$. Loss in mitochondrial membrane potential $\left(\psi_{\mathrm{m}}\right)$ was assessed as described previously ${ }^{19}$ by staining cells with TMRE, a lipophilic fluorescent dye that accumulates in the mitochondria in relation to the membrane potential, and quantified by flow cytometry. For BAK activation, cells were fixed with $2 \%$ paraformaldehyde at room temperature for $10 \mathrm{~min}$, washed with PBS and resuspended in a buffer containing $0.1 \%$ saponin and $0.5 \%$ BSA in PBS for $10 \mathrm{~min}$. The cell suspension was then incubated with $0.1 \mathrm{mg} / \mathrm{ml}$ of anti-BAK AB-1 (Calbiochem Research Biochemicals-now Merck, cat\#AM-03) antibody for $1 \mathrm{~h}$ at $4{ }^{\circ} \mathrm{C}$, followed by further incubation with goat-anti-mouse IgG-AlexaFluor-488 conjugated secondary antibody for $1 \mathrm{~h}$ at $4{ }^{\circ} \mathrm{C}$, before being subjected to flow cytometry.

\section{Statistical analysis}

Statistical analysis was conducted by using one-way ANOVA with Bonferroni's multiple comparison test was performed to evaluate differences between numerical variables. Asterisks depicted correspond to the following $p$ values: $" p \leq 0.05, * * p \leq 0.005$ and ${ }^{* * *} p \leq 0.001$.

\section{Acknowledgements}

We thank AbbVie for the BH3 mimetics and Drs. Youle, Clark, Slupsky and Bampton for the different cells and plasmids used in the study. This work was supported by a Science Without Borders Scholarship, CNPq 233624/2014-7, Ministry of Education, Brazil (to MM), studentship by Ministry of Higher Education and Scientific Research and University of Al-Qadisiyah, Iraq (to AA), NIH Grants R03CA205496 and R01GM118437 (to XL) and a North West Cancer Research Grant CR1040 (to SV and GMC).

\section{Author details \\ 'Department of Molecular and Clinical Cancer Medicine, Institute of Translational Medicine, University of Liverpool, Liverpool, Ashton Street, Liverpool L69 3GE, UK. ²Department of Cellular and Molecular Physiology, Institute of Translational Medicine, University of Liverpool, Liverpool, Ashton Street, Liverpool L69 3GE, UK. ${ }^{3}$ Eppley Institute for Research in Cancer and Allied Diseases, Fred and Pamela Buffett Cancer Center, University of Nebraska Medical Center, Omaha, NE 68198, USA. ${ }^{4}$ Department of Molecular and Clinical Pharmacology, Institute of Translational Medicine, University of Liverpool, Liverpool, Ashton Street, Liverpool L69 3GE, UK}

\section{Conflict of interest}

The authors declare that they have no conflict of interest.

\section{Publisher's note}

Springer Nature remains neutral with regard to jurisdictional claims in published maps and institutional affiliations.

The online version of this article (https://doi.org/10.1038/s41420-019-0199-x) contains supplementary material, which is available to authorised users.
Received: 5 June 2019 Revised: 17 June 2019 Accepted: 23 June 2019 Published online: 17 July 2019

\section{References}

1. Adams, J. M. \& Cory, S. The Bcl-2 apoptotic switch in cancer development and therapy. Oncogene 26, 1324-1337 (2007).

2. Danial, N. N. \& Korsmeyer, S. J. Cell death: critical control points. Cell 116, 205-219 (2004).

3. Youle, R. J. \& Strasser, A. The BCL-2 protein family: opposing activities that mediate cell death. Nat. Rev. Mol. Cell Biol. 9, 47-59 (2008).

4. Chen, L. et al. Differential targeting of prosurvival BCl-2 proteins by their BH3only ligands allows complementary apoptotic function. Mol. Cell 17, 393-403 (2005).

5. Lessene, G., Czabotar, P. E. \& Colman, P. M. BCL-2 family antagonists for cancer therapy. Nat. Rev. Drug Discov. 7, 989-1000 (2008).

6. van Delft, M. F. et al. The $\mathrm{BH} 3$ mimetic ABT-737 targets selective $\mathrm{BCl}-2$ proteins and efficiently induces apoptosis via Bak/Bax if Mcl-1 is neutralized. Cancer Cell 10, 389-399 (2006)

7. Tse, C. et al. ABT-263: a potent and orally bioavailable $\mathrm{BCl}-2$ family inhibitor. Cancer Res. 68, 3421-3428 (2008).

8. Souers, A. J. et al. ABT-199, a potent and selective BCL-2 inhibitor, achieves antitumor activity while sparing platelets. Nat. Med. 19, 202-208 (2013).

9. Leverson, J. D. et al. Exploiting selective BCL-2 family inhibitors to dissect cell survival dependencies and define improved strategies for cancer therapy. Sci. Transl. Med. 7, 279ra40 (2015).

10. Leverson, J. D. et al. Potent and selective small-molecule MCL-1 inhibitors demonstrate on-target cancer cell killing activity as single agents and in combination with ABT-263 (navitoclax). Cell Death Dis. 6, e1590 (2015).

11. Kotschy, A. et al. The MCL1 inhibitor $\mathbf{S 6 3 8 4 5}$ is tolerable and effective in diverse cancer models. Nature 538, 477-482 (2016).

12. Caenepeel, S. et al. AMG 176, a selective MCL1 inhibitor, is effective in hematologic cancer models alone and in combination with established therapies. Cancer Discov. 8, 1582-1597 (2018).

13. Tron, A. E. et al. Discovery of Mcl-1-specific inhibitor AZD5991 and preclinical activity in multiple myeloma and acute myeloid leukemia. Nat. Commun. $\mathbf{9}$, 5341 (2018).

14. Casara, P. et al. S55746 is a novel orally active BCL-2 selective and potent inhibitor that impairs hematological tumor growth. Oncotarget 9 , 20075-20088 (2018)

15. Roberts, A. W. et al. Targeting BCL2 with Venetoclax in relapsed chronic lymphocytic leukemia. N. Engl. J. Med. 374, 311-322 (2015).

16. Lucas, C. M. et al. High CIP2A levels correlate with an antiapoptotic phenotype that can be overcome by targeting $\mathrm{BCL}-\mathrm{XL}$ in chronic myeloid leukemia. Leukemia 30, 1273-1281 (2016).

17. Vaillant, F. et al. Targeting BCL-2 with the BH3 mimetic ABT-199 in estrogen receptor-positive breast cancer. Cancer Cell 24, 120-129 (2013).

18. Oltersdorf, $\mathrm{T}$. et al. An inhibitor of Bcl-2 family proteins induces regression of solid tumours. Nature 435, 677-681 (2005).

19. Vogler, M. et al. A novel paradigm for rapid ABT-737-induced apoptosis involving outer mitochondrial membrane rupture in primary leukemia and lymphoma cells. Cell Death Differ. 15, 820-830 (2008).

20. Henz, $K$. et al. Selective BH3-mimetics targeting BCL-2, BCL-XL or MCL-1 induce severe mitochondrial perturbations. Biol. Chem. 400, 181-185 (2019).

21. Hardwick, J. M., Chen, Y.-B. \& Jonas, E. A. Multipolar functions of BCL-2 proteins link energetics to apoptosis. Trends Cell Biol. 22, 318-328 (2012).

22. Chen, Y.-B. et al. BCl-XL regulates mitochondrial energetics by stabilizing the inner membrane potential. J. Cell Biol. 195, 263-276 (2011).

23. $\mathrm{Li}, \mathrm{H}$. et al. A BCl-xL-Drp1 complex regulates synaptic vesicle membrane dynamics during endocytosis. Nat. Cell Biol. 15, 773-785 (2013).

24. Perciavalle, R. M. et al. Anti-apoptotic MCL-1 localizes to the mitochondrial matrix and couples mitochondrial fusion to respiration. Nat. Cell Biol. 14, 575-583 (2012)

25. Varadarajan, S. et al. Sabutoclax (B|97C1) and Bl112D1, putative inhibitors of MCL-1, induce mitochondrial fragmentation either upstream of or independent of apoptosis. Neoplasia 15, 568-578 (2013).

26. Varadarajan, S. et al. Maritoclax and dinaciclib inhibit MCL-1 activity and induce apoptosis in both a MCL-1-dependent and -independent manner. Oncotarget 6, 12668-12681 (2015)

27. Milani, M. et al. DRP-1 is required for $\mathrm{BH} 3$ mimetic-mediated mitochondrial fragmentation and apoptosis. Cell Death Dis. 8, e2552 (2017). 
28. Hoppins, S., Lackner, L. \& Nunnari, J. The machines that divide and fuse mitochondria. Annu. Rev. Biochem. 76, 751-780 (2007).

29. Marín-García, J. \& Akhmedov, A. T. Mitochondrial dynamics and cell death in heart failure. Heart Fail. Rev. 21, 123-136 (2016).

30. Bertholet, A. M. et al. Mitochondrial fusion/fission dynamics in neurodegeneration and neuronal plasticity. Neurobiol. Dis. 90, 3-19 (2016).

31. Vogler, M., Dinsdale, D., Dyer, M. J. S. \& Cohen, G. M. ABT-199 selectively inhibits BCL2 but not BCL2L1 and efficiently induces apoptosis of chronic lymphocytic leukaemic cells but not platelets. Br. J. Haematol. 163, 139-142 (2013).

32. Al-Zebeeby A. et al. Targeting intermediary metabolism enhances the efficacy of $\mathrm{BH} 3$ mimetic therapy in haematological malignancies. Haematologica. 104, 1016-1025 (2019).

33. O'Neill, K. L., Huang, K., Zhang, J., Chen, Y. \& Luo, X. Inactivation of prosurvival $\mathrm{BCl}-2$ proteins activates Bax/Bak through the outer mitochondrial membrane. Genes Dev. 30, 973-988 (2016).

34. Greaves, G. et al. BH3-only proteins are dispensable for apoptosis induced by pharmacological inhibition of both $\mathrm{MCL}-1$ and BCL-XL. Cell Death Differ. 26 1037-1047 (2019).

35. Scorrano, L. et al. A distinct pathway remodels mitochondrial cristae and mobilizes cytochrome c during apoptosis. Dev. Cell 2, 55-67 (2002).

36. Morciano, G. et al. Mcl-1 involvement in mitochondrial dynamics is associated with apoptotic cell death. Mol. Biol. Cell 27, 20-34 (2016).
37. Prudent, J. et al. MAPL SUMOylation of Drp1 Stabilizes an ER Mitochondrial Platform Required for Cell Death. Mol. Cell 59, 941-955 (2015).

38. Wang, P. et al. Dynamin-related protein Drp1 is required for Bax translocation to mitochondria in response to irradiation-induced apoptosis. Oncotarget $\mathbf{6}$, 22598-22612 (2015).

39. $\mathrm{Xu}, \mathrm{W}$. et al. Bax-PGAM5L-Drp1 complex is required for intrinsic apoptosis execution. Oncotarget 6, 30017-30034 (2015).

40. Lee, J. E., Westrate, L. M., Wu, H., Page, C. \& Voeltz, G. K. Multiple dynamin family members collaborate to drive mitochondrial division. Nature 540, 139-143 (2016).

41. Friedman, J. R. et al. ER tubules mark sites of mitochondrial division. Science 334, 358-362 (2011).

42. Cho, B. et al. Constriction of the mitochondrial inner compartment is a priming event for mitochondrial division. Nat. Commun. 8, 15754 (2017).

43. Otera, H., Miyata, N., Kuge, O. \& Mihara, K. Drp1-dependent mitochondrial fission via MiD49/51 is essential for apoptotic cristae remodeling. J. Cell Biol. 212, 531-544 (2016).

44. Yamaguchi, R. et al. Opa1-mediated cristae opening is Bax/Bak and BH3 dependent, required for apoptosis, and independent of Bak oligomerization. Mol. Cell 31, 557-569 (2008).

45. Vogler, $M$. et al. Concurrent up-regulation of $B C L-X L$ and $B C L 2 A 1$ induces approximately 1000-fold resistance to ABT-737 in chronic lymphocytic leukemia. Blood 113, 4403-4413 (2009). 